\title{
Las Competencias Investigativas al Servicio de las Competencias Gerenciales
}

\section{The Investigative Competences at the Service of Management Competences}

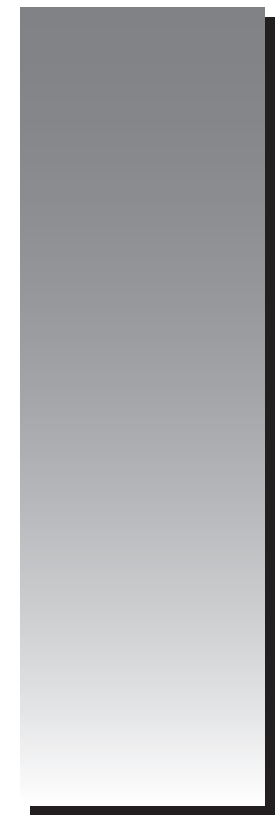

Patricia Hidalgo Euribe ${ }^{1}$ phidalgovel@gmail.com

\section{Armando Velazco Ríos ${ }^{2}$}

Avelazcor@ci.edu.pe

Universidad Nacional Mayor de San Marcos

Palabras claves: Competencias gerenciales; competencias investigativas; cursos de investigación; desarrollo de tesis.

\begin{abstract}
The postgraduate specializations in universities of the country and abroad incorporate in their curriculum mesh the research courses that, under the name of Methodology of the scientific investigation, Seminar or Research Workshop, pretend, for example, develop the investigative competences in the students and, on the other hand, that these achieve advance as much as possible in the different stages of construction of the thesis that allow to sustain it and obtain the academic degree. The progress of the test is based on the fact that the objectives can be easily identified, the embargo, the weight of its importance, is not always clear, it answers the question that the research competencies that are intended to be developed and those that are not They are in due they are the impact of these courses in the generation of professional competences of the student. We will try to answer the question referring in particular to the specializations in Administrative Sciences and Management.
\end{abstract}

Keywords: Management skills; investigative skills; research courses; thesis development.

\footnotetext{
1 Doctora en Administración por la Universidad Nacional Mayor de San Marcos. Magister en Gerencia Social y Licenciada en Trabajo Social por la Pontificia Universidad Católica del Perú, Diplomada en Antropología por la misma universidad. Estudios de Especialización en Dirección Estratégica en CENTRUM. Docente de la Universidad Nacional Mayor de San Marcos en la Facultad de Ciencias Administrativas en las menciones de Gestión Empresarial, Gestión de Recursos Humanos y Gestión Pública; ha sido Coordinadora ejecutiva y docente en la Maestría en Gerencia Social de la Pontificia Universidad Católica del Perú. Asesora y jurado de Tesis de maestría y doctorado. Consultora en investigación aplicada en gestión organizacional y programas para el desarrollo.

2 Egresado del Programa de Doctorado en Ciencias Administrativas de la Universidad Nacional Mayor de San Marcos, Magister en Administración de la Universidad del Pacífico y Contador Público de la Universidad Nacional Mayor de San Marcos.
}

(C) Los autores. Este artículo es publicado por Gestión en el Tercer Milenio de la Facultad de Ciencias Administrativas de la Universidad Nacional Mayor de San Marcos. Este es un artículo de acceso abierto, distribuido bajo los términos de la licencia Creative Commons Atribucion - No Comercia_Compartir Igual 4.0 Internacional. (http://creativecommons.org/licenses/by-nc-sa/4.0/) que permite el uso no comercial, distribución y reproducción en cualquier medio, siempre que la obra original sea debidamente citada. 


\section{INTRODUCCIÓN}

Quienes nos desempeñamos en el mundo académico somos testigos de las dificultades que los estudiantes enfrentan para concluir el proceso de elaboración de la tesis. Para ello deberán transitar por varios cursos de investigación, de modo que progresivamente vayan cerrando etapas desde un inicial plan de tesis hasta su redacción y sustentación final.

Una interrogante que resulta central está referida al impacto de estos cursos en la generación de competencias profesionales del estudiante. Es evidente que los cursos se encuentran estructurados a partir de competencias, pero, ¿qué sucede en el camino?, ¿La formación en investigación se lleva a cabo realmente como un proceso intencional para el desarrollo de competencias o básicamente para la culminación de la tesis u obtención del grado?, ¿Las asignaturas pierden en algún momento esta importante finalidad?

Aunque esta situación pareciera ser común en postgrados de diversas especialidades, centraremos esta preocupación en aquellos dirigidos a formar en competencias gerenciales, preguntándonos en qué medida las competencias investigativas colaboran o forman parte de las competencias gerenciales para el desempeño profesional.

Si bien podemos considerar a priori que las competencias gerenciales se ven mejoradas y fortalecidas con el desarrollo de las competencias investigativas, se hace necesario desarrollar dicha idea.

\section{OBJETIVO DEL ENSAYO}

Esta preocupación quiere estar en la línea de aquellos que advierten una evolución en la formación para la investigación, según la cual se pretende pasar de la obtención de contenidos al desarrollo de habilidades y actitudes y lograr con ello el saber, saber hacer y saber ser.

En esa línea, la principal inquietud es cómo contribuir, desde los cursos de investigación de las Facultades de Administración, principalmente de postgrado, en la formación de profesionales con competencias investigativas que aporten al ejercicio de las competencias ger- enciales al interior de sus organizaciones para resolver problemas, sean estos de nivel estratégico, programático o táctico; aplicados a la viabilidad económica y financiera de proyectos o a los recursos humanos.

Empezaremos entonces dedicando algunas líneas a ubicar cuál es el espacio que se le da a la investigación en las organizaciones en que se desempeñan los profesionales para de ahí pasar a señalar que rasgos de las competencias y habilidades investigativas complementan y aportan a las competencias gerenciales, propias del rol y desempeño de los gerentes.

\section{Las Competencias Investigativas y las Competencias Gerenciales}

Resulta claro que las organizaciones están envueltas en un entorno aceleradamente cambiante; y para ello adaptarse y generar procesos y estrategias para crecer, competir y desarrollarse, constituye un reto. En ello la generación de conocimiento para el progreso a un nuevo estado organizacional resulta un factor clave.

En este espacio organizativo, tienen cabida los gerentes y especialistas de diversas disciplinas pues todos al final forman parte de un mismo sistema. Este desarrollo del pensamiento administrativo está presente no solo en grandes organizaciones, sino también en medianas y pequeñas. Por lo mismo se hace necesaria una formación para conceptualizar los problemas y transformar la información en conocimiento.

La investigación científica es una manera de generar conocimiento, la cual, como señala Hernández (2014) “...esta puede cumplir dos propósitos: a) Producir Conocimientos y teorías mediante la investigación básica y, b) Resolver problemas prácticos con la investigación aplicada".

Por la misma naturaleza de la administración como disciplina social que trata de dar respuestas permanentes a un entorno cambiante, la investigación científica básica es escasa en tanto ella no busca establecer nuevas leyes o teorías, sino más bien la resolución de los problemas corrientes a partir de investigaciones aplicadas que permitan establecer políticas y estrategias de largo plazo. 
Como dice Duane (2001) al referirse a la necesidad de fundamentar las decisiones:

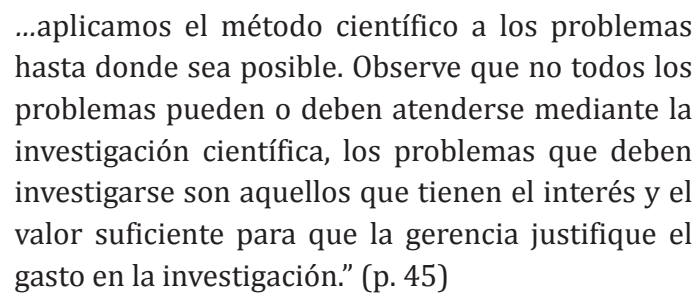
hasta donde sea posible. Observe que no todos los problemas pueden o deben atenderse mediante la investigación científica, los problemas que deben investigarse son aquellos que tienen el interés y el valor suficiente para que la gerencia justifique el gasto en la investigación." (p. 45)

El mismo autor hace un llamado a los gerentes e investigadores a no detenerse demasiado en la disquisición si el estudio se considera científico o no "Lo que sí importa es que mantengamos una percepción clara de las necesidades de información de la gerencia y cómo podemos satisfacerlas mejor". (Duane, 2001, p.38). Cuando los problemas son únicos y no repetitivos son los gerentes de niveles superiores quienes van a lidiar con este tipo de decisiones inusuales y no rutinarias. Pero también hay decisiones que suelen ser programadas, es decir se conoce la regularidad de los hechos -en cuyo caso hay ya reglas establecidas- y pueden ser resueltos a partir de la Investigación de 0peraciones, la cual tiene larga data en la historia de la administración con un campo muy amplio pues permite implementar métodos o herramientas que van a conducir de manera concreta y operativa a tomar opciones a partir de la información que se suministra.

En el lenguaje académico y profesional, el término "competencias" viene siendo la mejor manera de referirnos a aquello que como conocimientos, habilidades, destrezas y actitudes se debe disponer para realizar en forma eficiente y eficaz una labor; es decir un saber actuar. El énfasis en las competencias, más que en los conocimientos adquiridos, se da a partir de la década de los noventa con las nociones referidas a la gestión del talento humano.

Tobón (2013), estudioso del enfoque complejo de las competencias en educación, y en especial en educación superior, menciona que hay diversos enfoques para abordar las competencias debido a las múltiples fuentes, perspectivas y epistemologías que han estado implicadas en el desarrollo de este concepto, así como en su aplicación tanto en la educación como en las organizaciones señalando la importancia de una transformación curricular y una propuesta de formación integral.
¿Por qué referirnos en el campo administrativo a las competencias que se desarrollan a partir de la investigación científica? Precisamente porque estas van a contribuir al ejercicio de las funciones gerenciales que se ponen en juego "como un conjunto de conocimientos, destrezas, comportamientos y actitudes que necesita una persona para ser eficiente en una amplia gama de labores gerenciales en diversas organizaciones " (Hellriegel, Jackson y Slocum ,2016, p.5)

Campos y chinchilla (2009) señalan que las competencias gerenciales se desarrollan idealmente a lo largo de la formación en su conjunto y Moreno (2005) refiere que las competencias básicas para la investigación es un trabajo de carácter sistémico de largo plazo. En ese sentido, los cursos de investigación en las universidades juegan un rol fundamental en la consolidación no solo de los conocimientos necesarios, sino de una actitud de confianza que le permita al profesional tener un alto desempeño al enfrentar los desafíos del mundo laboral y social.

A decir de Hellriegel, Jackson y Slocum (201, p.5) son 6 las competencias gerenciales claves para: la planeación y gestión, la acción estratégica, la comunicación, la multiculturalidad, el trabajo en equipo, el autocontrol y como bien lo señalan, en la práctica resulta difícil saber dónde empieza una y termina la otra.

En los siguientes acápites procuraremos descubrir en qué aspectos las competencias investigativas pueden sumarse a las competencias gerenciales mencionadas, para lo cual privilegiaremos las que consideramos que tienen una mayor pertinencia en relación al tema de este artículo.

\section{1. ¿Qué competencias investigativas pueden afianzar las competen- cias gerenciales de PLANEACIÓN Y GESTIÓN?}

La competencia para la planeación y administración "comprende, decidir qué áreas hay que realizar, determinar la manera de efectuarlas, asignar los recursos que permitan llevarlas a cabo y, luego supervisar la evolución para asegurarse de que se hagan (...) Esta competencia comprende entre otros puntos: Recopilar y analizar información y resolver problemas; Pla- 
near y organizar proyectos; Administrar el tiempo, y; Presupuestar y administrar las finanzas". (Hellriegel, Jackson y Slocum, 2016, p.16). En cuanto a las competencias investigativas que pueden aportar a la planeación y gestión, proponemos las siguientes:

\section{Saber identificar el problema principal.}

¿Formular la pregunta principal o pregunta de investigación no es acaso el primer paso para plantear el problema y el inicio de la identificación de alternativas para la toma de decisiones? Es importante caer en la cuenta de lo relevante que resulta plantearse interrogantes, lo cual permite a su vez predecir de cierta forma cuales podrían ser sus respuestas, contextualizándolas, determinando su prioridad, magnitud, dimensión y trascendencia.

Durante la formación de los estudiantes son los docentes quienes enfrentan el reto de colaborar con ellos en la formulación creativa de interrogantes de relevancia y en una línea de innovación. Asumir la complejidad de la realidad y la integralidad de los problemas para concretizar la formulación en una pregunta que recoja la perspectiva bajo la cual se analiza, se soporta en un conjunto de operaciones mentales necesarias para ello.

Los cursos de investigación en las maestrías y doctorados parten del supuesto que los estudiantes saben de antemano donde identificar los problemas, y si bien esto depende de su experiencia, de su interés temático y de las posibilidades de realización en función a su ubicación actual en la organización, nos podemos preguntar ¿realmente saben dónde identificar los problemas?

En un primer momento del proceso de investigación se identificará un conjunto de problemas relacionados para pasar luego a priorizar e identificar el problema principal y con ello disgregar los problemas secundarios, es decir significa estar en la capacidad de diferenciar los aspectos centrales de los que no lo son, como las causas y efectos que configuran la situación problemática y que permite presentarla en su complejidad.

¿Qué competencias gerenciales se refuerzan en esta etapa? Es posible desarrollar las competencias y habilidades vinculadas a las habilidades básicas cognoscitivas (observar, relacionar, describir entre otras) así como las de pensamiento crítico y reflexivo. A decir de Ander Egg (1983, p.50) se desarrollan operaciones mentales de representación y de identificación de problemas como: enumerar y describir, comparar y distinguir, clasificar y definir, buscar contradicciones y oposiciones, identificar aspectos y puntos de vista así como saber situar los hechos y fenómenos en el tiempo y en el espacio.

Se trata entonces de comprender y describir el problema en su relación entre variables, las causas y efectos que ocasiona, los actores involucrados y la prioridad que estos tienen en el entorno organizativo, de esta manera se desarrollan las condiciones para efectuar un análisis bajo un pensamiento crítico, lógico y reflexivo.

Poner en práctica las habilidades de construcción metodológica.

El diseño de procedimientos e instrumentos permitirá el recojo de la data para sistematizar y analizar la información. Aún cuando no sean los gerentes los encargados directos, ya que pueden ser asignados a otros colaboradores o terceros, siempre será importante contar con las competencias para saber qué y donde encontrar la información, cuáles son los instrumentos adecuados para ello y principalmente si hay coherencia entre lo esperado y la información obtenida (variables-indicadores-instrumentos).

\section{Analizar la información}

Es una competencia cognitiva básica en investigación de forma tal que la búsqueda de información relevante y su uso eficiente contribuyan a decisiones específicas y oportunas. La data recogida se presenta en evidencias gráficas, cuantificables, estadísticas y también de naturaleza cualitativa.

La competencia para seleccionar información supone estar actualizado con el uso de la tecnología de la información, así como en nuevos y complejos métodos de análisis de datos; siendo evidente que es posible asociar los cambios en la búsqueda de información con la manera en la que se hace investigación (Duane, 2001). 
La accesibilidad en tiempo real es decir su oportunidad y utilidad, su procesamiento a partir de sistemas, la posibilidad de análisis inmediato no solo de fuentes internas sino también externas es desde ya un recurso importante. Así, aquello que parece habitual y sencillo como es buscar información requerirá de algunos niveles de experticia importantes, siendo necesario considerar "la forma en que las tecnologías pasan a potenciar los procesos cognitivos a través de una experiencia que también abona en lo emocional, creativo y dialógico" (Navarro, Aguirre y Balderrama 2016, p.56).

Esta competencia se desarrolla durante los cursos de investigación en la medida que prácticamente todo el proceso se ve acompañado por la necesidad de indagar sobre antecedentes, avances y perspectivas actuales sobre el tema y problema de investigación es decir la construcción del llamado "estado del arte".

¿Qué buscar y dónde buscar? Son preguntas que deben ir acompañadas de las habilidades para indagar y obtener información de fuentes certeras, y por consiguiente los cursos de investigación debieran preparar para ello, de manera que las plataformas educativas, la consulta de Blog, software especializados, las webs temáticas sean utilizados en la perspectiva de contribuir, tanto - cuanto, a la búsqueda de información.

\section{2. ¿Qué competencias investigativas pueden afianzar las competencias gerenciales para la ACCIÓN ES- TRATÉGICA?}

A este nivel la competencia gerencial para la acción estratégica está vinculada a la toma de decisiones que se sustentan en la identificación acertada de situaciones problemáticas y de alternativas sobre las cuales se determinan estrategias y planes de acción lo suficientemente claros y precisos como para lograr el desarrollo de la organización y obtener una ventaja competitiva.

“Entender la misión y los valores generales de la organización y asegurarse de que las acciones propias y las de quienes uno dirige están alineadas es algo que exige competencias en la acción estratégica, la cual comprende: entender la industria, comprender la organización, adoptar medidas estratégicas" (Hellriegel, Jackson y Slocum, 2016, p.16).

\section{Contar con una estrategia metodológica}

En términos generales, así como en el proceso de investigación se decide la estrategia metodológica, o los criterios de selección de la muestra; así en las organizaciones es posible asumir como forma de aproximarse al conocimiento la realización de investigaciones aplicadas, de manera que sus resultados permitan un accionar estratégico.

Como ya se señaló, enfrentarse a la solución de un problema, no es otra cosa que asumir decisiones en torno a cambios organizacionales, mejoras en las estrategias, priorización de tareas específicas, etc., y todo ello en la búsqueda de las mejores decisiones de las que dependen en buena medida la marcha de la organización.

\section{3. ¿Qué competencias investigativas pueden afianzar las competencias gerenciales para LA COMUNICACIÓN?}

La comunicación es la capacidad para transmitir e intercambiar eficazmente información para entenderse con los demás, sea esta de modo formal o informal. Es parte importante de la formación profesional, en términos de una habilidad instrumental, el dominio formal del lenguaje que le permitirá la presentación eficiente de sus trabajos y conclusiones.

\section{Defender consistentemente las ideas}

Las relaciones con los demás para presentar y defender ideas se ponen en juego desde la presentación y defensa en las aulas de las ideas iniciales que van luego tomando forma de proyecto de investigación. No son pocas las veces que los estudiantes se enfrentan a la exposición de ideas durante los estudios de especialización, sin embargo, no hay nada más propio y particular a ser defendido que la propuesta de investigación. En cada curso de investigación, seminarios o talleres de tesis las exigencias de hilvanar los contenidos teóricos los coloca en la necesidad de defender en forma solvente su planteamiento.

La rigurosidad del método y las exigencias de consistencia interna y coherencia ex- 
igen precisamente poner en juego una de las competencias gerenciales más importantes: La comunicación.

Esta competencia de saber comunicar los acompañará a lo largo de su vida profesional en todas aquellas ocasiones en que se tenga que exponer ideas, propuestas y/o proyectos. En todos los casos la posibilidad de entendimiento con sus interlocutores, y por consiguiente de aceptación, pasa por la claridad con que sean transmitidos.

\section{CONCLUSIONES}

Los cursos de investigación en los programas de especialización en las universidades colaboran en el desarrollo de las competencias gerenciales, en este sentido es que el artículo pone en consideración que más allá de iniciar y culminar el proceso de desarrollo de la tesis de grado - ciertamente importante - se pretende que los estudiantes hagan suyas las competencias investigativas que posteriormente les permitirá ejercer las competencias gerenciales de manera efectiva.

Las organizaciones son un campo amplio en el que es posible desarrollar investigaciones aplicadas en el que los gerentes pueden y deben participar.

Dichas competencias investigativas son requeridas para el ejercicio de las funciones profesionales en el ámbito organizacional, que deben contribuir a la solución de problemas que todo gerente debe saber identificar, priorizar y abordar en vistas a tomar decisiones.

\section{BIBLIOGRAFÍA}

Ander Egg, Ezequiel (1983). Técnicas de Investigación Social. Décimo cuarta edición. Gráfica Diaz, S.L. Alicante

Campos, J y Chinchilla, A (2009). Reflexiones acerca de los desafíos en la formación de competencias para la investigación en educación superior.

Chiavenato, Idalberto (2006). Introducción a la teoría general de la administración. Séptima edición. Mc Graw Hill Interamericana, México.

Duane, David (2001). Investigación en Administración. México: Thomson Editores.

Kast, F \& Rosenzweis, J. (1988) Administración en las Organizaciones: Enfoque de Sistemas y de Contingencias. 4ta Edición, Mc Graw Hill, México.

Hellriegel, Jackson y Slocum (2016). Administración: un enfoque basado en competencias. 11 ava. Edición. Thomson learnig S.A. México.

Hernández, R, Fernández, R y Baptista, P (2014) Metodología de la Investigación Científica. 6ta. Edición. Mc Graw Hill Interamericana, México.

Hidalgo, Patricia y Tovar, T (2010). Material para el curso de Métodos y Técnicas de Investigación. Maestría en Gerencia social. PUCP. Perú.

Navarro, R., Aguirre, G y Balderrama, J. La contribución didáctica de las TICs en el desarrollo de competencias para la investigación. En: revista Ideales, Ibagué, Universidad del Tolima, Vol. 1, 2016, pp. $54-59$

Robbins, S y Judge, T (2010). Comportamiento organizacional. Decimocuarta edición. Pearson Educación de México. México.

Tobón, Sergio (2013). Dirección integral y competencias: pensamiento complejo, circulo, didáctica y evaluación. 4ta. Edición. Ecoe Ediciones, México. 